\title{
BIBLIOTECAS DE LOS COLEGIOS PROFESIONALES EN EL ÁREA DE SALUD EN CATALUÑA: SERVICIOS ACTUALES Y NECESIDADES DE ACTUACIÓN .
}

\section{Eva Ortoll Espinet*}

Resumen: Las tendencias anglosajonas ponen de manifiesto la importancia de
la acreditación de profesionales a través de los colegios profesionales, con
especial incidencia en el papel que las bibliotecas deben desarrollar como di-
fusores de servicios de información y especialmente de información biblio-
gráfica. Se analizan los servicios que ofrecen las bibliotecas de los colegios
profesionales relacionados con el ámbito de la salud en Cataluña mediante
la utilización de una encuesta de opinión. Los resultados demuestran la
necesidad de potenciar su uso y de la elaboración de unas pautas que sir-
van para la creación de unos servicios mínimos que deberían ofrecer las
bibliotecas de los colegios profesionales relacionados con cualquier disci-
plina.

Palabras clave: bibliotecas, servicios de información, colegios profesionales.

\begin{abstract}
The recent importance of professional accreditation through professional associations is increasing every day. Professional associations must provide information and services to their members, with especial attention on the library services. This paper makes an analysis of the services offered by professional associations libraries, related to biomedical and health sciences in Catalonia. The method employed has been a questionnaire. The results obtained show the need to improve the services and their use, and the importance of developing some recommendations in order to help the construction of a minimum number of services that should be offered by these institutions.
\end{abstract}

Keywords: libraries, information services, professional associations.

\section{Introducción}

La importancia de facilitar el acceso a la información científica en el ámbito de las ciencias de la salud ha estado ampliamente discutida en la literatura científica; esta necesidad se ve acrecentada por las repercusiones que en la práctica clínica puede llegar a tener dicha información (1). Los colegios profesionales constituyen una plataforma importante para sus miembros, el papel que pueden jugar como centro canalizador y difusor de información laboral, profesional y también bibliográfica, así como punto clave en la acreditación de sus profesionales (2-3), nos lleva a plantearnos la necesidad de estudiar la situación actual de las bibliotecas de los colegios profesionales relacionados con los distintos colectivos cuyo ámbito de actividad sean las ciencias de la salud.

Otros estudios realizados sobre la situación de las bibliotecas de ciencias de la sa-

* Universidad Rovira i Virgili. Biblioteca de Medicina, Reus (Tarragona). Correo-e: bibmed(a) astor.urv.es Recibido: 1." versión: 14-6-99; 2." versión: 23-3-00. 
lud en España (4) no incluyen, por motivos de operatividad, y discriminando otras tipologías de centros susceptibles de ser analizadas, los colegios profesionales. Por ello nos hemos propuesto completar los trabajos existentes con el análisis de dichos centros.

El estudio realizado pretende ser una prueba piloto que sirva de base para un análisis posterior; la limitación geográfica será Cataluña. Esperamos que los resultados obtenidos nos permitan modificar, ampliar, mejorar y elaborar un plan de trabajo más extenso, con el objetivo de realizar un estudio exhaustivo, que abarque la totalidad de colegios profesionales relacionados con las ciencias de la salud existentes en las distintas provincias españolas.

\section{Metodología}

\subsection{Población de estudio}

Estudio descriptivo utilizando una encuesta de opinión sobre la totalidad de la población objeto de estudio. La población inicial del estudio la componen los colegios oficiales de aquellos colectivos directamente relacionados con el ámbito de las ciencias de la salud; identificando inicialmente como tales los colegios de médicos, farmacéuticos, diplomados en enfermería, odontólogos y fisioterapeutas.

La cobertura geográfica del estudio se limita a la Comunidad Autónoma de Cataluña, por los motivos expuestos en la introducción.

Se excluyen los restantes centros de este ámbito (universidades, hospitales, etc.) por haber sido ya objeto de estudio en anteriores trabajos (5-6).

\subsection{Identificación de la población inicial de estudio}

Los datos que han permitido identificar y obtener las direcciones de la población de estudio se han obtenido consultando directorios electrónicos o impresos' ${ }^{\prime}$. Finalmente se contó con la colaboración del Col-legi de Metges de Tarragona que nos facilitó las direcciones no localizadas en los directorios consultados.

Con este proceso de búsqueda se identificaron 14 centros de los 20 inicialmente previstos (5 por provincia); dado que de los colegios de fisioterapeutas y odontólogos no existe una sede por provincia, se engloban los cuatro en un colegio único para toda la Comunidad Autónoma.

De los 14 centros que deberían configurar el directorio, se incluyen únicamente 10 al no disponer los otros de ningún servicio y/o atención a los usuarios que ejerza la función de unidad de información o no responder a la encuesta.

Los centros a los que se envió la encuesta fueron:

- Col-legi Oficial de Metges de Barcelona.

- Col·legi Oficial de Metges de Tarragona.

\footnotetext{
'Quién es quién en Información y Documentación y Bibliotecas. Madrid: EBSCO, 1998.

Organización Médica Colegial. Colegios Médicos Provinciales en URL: http://www.telprof.es/omc/
} 
- Col.legi Oficial de Metges de Girona.

- Col-legi Oficial de Metges de Lleida.

- Col-legi Oficial de Farmacèutics de Barcelona.

- Col-legi Oficial de Farmacèutics de Tarragona.

- Col-legi Oficial de Farmacèutics de Girona.

- Col-legi Oficial de Farmacèutics de Lleida.

- Col-legi Oficial de Diplomats en Infermeria de Barcelona.

- Col-legi Oficial de Diplomats en Infermeria de Tarragona.

- Col-legi Oficial de Diplomats en Infermeria de Lleida.

- Col-legi Oficial de Diplomats en Infermeria de Girona.

- Col-legi Oficial de Fisioterapèutes de Catalunya.

- Col·legi Oficial d'Odontòlegs i Estomatòlegs de Catalunya.

\subsection{Recogida de datos y elaboración del cuestionario}

Se envió la encuesta siempre que fue posible mediante correo electrónico; en otros casos, y considerando factores como la ausencia de cuenta propia de los responsables de dichas unidades, se envió la encuesta por correo tradicional.

A pesar de ser pocos los centros estudiados, no todos tenían buena predisposición para responder el cuestionario. El total de respuestas obtenidas fue de 10; 2 de los centros restantes no disponían de unidad de información y los otros 2 no contestaron al cuestionario.

Los resultados de esta primera recogida servirán para modificar aquellas preguntas del cuestionario que sean confusas, innecesarias o sin contestar en un $90 \%$ de los encuestados. La finalidad es poder trabajar con una prueba piloto de lo que pretende ser un estudio a escala estatal.

El cuestionario se elaboró a partir del modelo utilizado en el proyecto de investigación de Isabel Ubieto Artur (7) sobre el Sistema de Información Aragonés.

\section{Resultados}

\subsection{Colección}

Volumen de la Colección

\section{- Monografías}

5 centros cuentan con colecciones inferiores a 300 volúmenes, 2 con colecciones entre 500 y 1500 volúmenes y 3 con colecciones entre 2000 y 5000 volúmenes.

\section{- Otro material}

La inclusión de distintos tipos de materiales en las colecciones es poco representativa. En los centros donde se especifica dicho material destaca la presencia en las colecciones de CD-ROM, con una media de 18 ítems. 


\section{- Tesis doctorales}

Especificadas únicamente en dos encuestas, representando el $0,7 \%$ de la colección en un caso y un $0,1 \%$ en el otro.

\section{- Revistas especializadas}

De los 10 centros analizados, 8 reciben revistas especializadas. En las colecciones mayores el número de títulos activos se sitúa alrededor de los 250 títulos, dos centros disponen de colecciones pequeñas entre 20 y 25 títulos y los restantes reciben publicaciones puntuales en número no superior a 5 .

\section{- Publicaciones oficiales} les.

De forma generalizada todos los centros reciben entre 2 y 4 publicaciones oficia-

\section{- Bases de datos}

El acceso a bases de datos contratando servidor externo se da en 3 casos, 1 de ellos tiene su propia intranet, 2 centros acceden a Medline u otras bases de datos en CDROM con acceso local y 2 de los centros sólo acceden a bases de datos gratuitas a través de Internet. Los centros restantes no ofrecen este servicio.

\section{Tabla I}

Bases de datos por número de centros

\begin{tabular}{|l|c|}
\hline \multicolumn{1}{|c|}{ Bases de datos } & Número de centros \\
\hline MEDLINE & 5 \\
CINHAL & 4 \\
IME & 4 \\
EMBASE & 3 \\
CURRENT CONTENTS & 1 \\
\hline
\end{tabular}

\section{- Cobertura cronológica e idiomática de la colección}

En un $65 \%$ de los casos, los fondos de las bibliotecas inician la cobertura cronológica en 1940. Excepcionalmente 2 centros disponen de obras anteriores a 1900. Por lo que se refiere al idioma engloban obras en catalán, español, francés e inglés en un $75 \%$ de los centros. Un $25 \%$ de ellos incluye en su colección obras en otros idiomas tales como italiano y alemán.

\subsection{Procesos técnicos}

\section{- Catálogos automatizados}

Sólo 5 centros disponen de catálogos automatizados, sólo uno de ellos con posibilidad de consulta a través de Internet. Los restantes catálogos automatizados son de consulta local en el propio centro. 


\section{- Catálogos manuales}

Los centros que disponen de catálogos manuales son 2.

\section{- Catálogo de publicaciones periódicas}

Disponen de catálogo de publicaciones periódicas impreso e informatizado 5 centros, 4 de ellos únicamente impresos.

\section{- Elaboración de documentos propios}

En un 55\% de los centros elaboran revistas de difusión del propio colegio, 4 casos; informes de actos y actividades organizados por los mismos, 5 , y trabajos de investigación, 8.

- Procesos que se aplican a los documentos

Tabla II

Procesos por número de centros

\begin{tabular}{|l|c|}
\hline \multicolumn{1}{|c|}{ Procesos } & Número de centros \\
\hline Catalogación & 5 \\
Clasificación & 6 \\
Revisión & 3 \\
Expurgo & \\
Resumen & 2 \\
Listados & 4 \\
\hline
\end{tabular}

\section{- Directrices utilizadas}

Un $10 \%$ de los centros utilizan reglas de catalogación (AACR) y un $25 \%$ la CDU como sistema de clasificación. En 4 centros elaboran catálogos de autores, materias y títulos, 2 incluyen el catálogo por clasificación y 1 el catálogo diccionario.

Por lo que se refiere a la utilización de tesauros o listas de encabezamientos, un $25 \%$ de los casos utiliza el generado por el propio programa, un $25 \%$ utiliza el tesauro informatizado de la Universidad de Barcelona. El 50\% restante no responde a esta pregunta.

\subsection{Servicios y usuarios}

\section{Tabla III}

Servicios por número de centros

\begin{tabular}{|l|c|}
\hline \multicolumn{1}{|c|}{ Servicios } & Número de centros \\
\hline Búsquedas bibliográficas & 8 \\
Búsquedas asistidas & 7 \\
Servicio de referencia & 3 \\
Difusión Selectiva de Información & 1 \\
Obtención del documento & 5 \\
Préstamo interbibliotecario & 4 \\
Servicio de publicaciones & 1 \\
Formación de usuarios & 1 \\
Préstamo individualizado & 5 \\
\hline
\end{tabular}




\section{- Préstamo individualizado}

En 3 centros el préstamo se restringe a los colegiados, 2 de ellos amplían el préstamo a los estudiantes universitarios que tienen algún contacto con el colegio. Los centros restantes no contemplan dichos servicios.

\section{- Consultas de usuarios}

En un $90 \%$ de los centros las peticiones de los usuarios se alternan utilizando el teléfono y contacto personal con el responsable de la unidad, un $25 \%$ atiende también solicitudes por fax y correo electrónico y 1 centro exclusivamente atiende todas las peticiones personalmente.

\section{- Acceso a la información}

En todos los centros el acceso a la información se realiza entre un $90 \%$ y un $100 \%$ de los casos a través del documentalista. Únicamente un centro ofrece cursos de formación de usuarios.

\section{- Gratuidad de los servicios}

Los centros que tarifan sus servicios son 3, los precios varían entre 10 a 20 ptas. la fotocopia, en los casos en los que se solicita la información al exterior se incrementa en un $0-15 \%$ el precio de coste del centro servidor externo.

\subsection{Cooperación}

\section{Tabla IV}

\section{Cooperación por número de centros}

\begin{tabular}{|l|c|}
\hline \multicolumn{1}{|c|}{ Tipo de cooperación } & Número de centros \\
\hline Intercambio de publicaciones & 3 \\
Intercambio de datos & 1 \\
Préstamo & 4 \\
Gestión de duplicados & 0 \\
Participación en Catálogos Colectivos & 3 \\
\hline
\end{tabular}

\subsection{Equipamiento y software de trabajo}

De los 10 centros analizados, 5 disponen de un PC/Pentium dedicado exclusivamente a la gestión del fondo documental. En todos esos casos, ese mismo ordenador se destina al punto de consulta de los usuarios. En los 5 centros restantes no se dispone de infraestructura propia y los recursos son compartidos con otros servicios del colegio.

El sistema de gestión de bases de datos utilizado para gestionar la biblioteca en 3 casos es el programa INMAGIC; en los centros restantes no se especifica. 


\subsection{Conexión a Internet}

Excepto 2 centros, los 8 restantes disponen de conexión a Internet.

\subsection{Personal del servicio}

\section{Tabla V}

Personal por número de centros

\begin{tabular}{|l|c|}
\hline \multicolumn{1}{|c|}{ Tipo de personal } & Número de centros \\
\hline Diplomados en Biblioteconomía & 2 \\
Auxiliares administrativos & 6 \\
Sin especificar & 2 \\
\hline
\end{tabular}

\section{Discusión}

El objetivo inicial del estudio identificaba 20 centros que potencialmente podrían disponer de una biblioteca o unidad de información. Tal como se ha expuesto en la metodología, el número de centros reales a estudiar se ha reducido a 14, 2 de los cuales no disponían de biblioteca o centro de documentación, y los 2 restantes no han respondido al cuestionario enviado.

Los 10 centros que se incluyen en el estudio disponen de biblioteca, unidad de información o servicio de atención para facilitar información bibliográfica a sus colegiados. Los criterios de inclusión para el análisis de los centros hubieran podido ser más selectivos; por ejemplo, considerar sólo aquellos centros que dispusieran de una colección organizada de libros y revistas y ofrecieran unos servicios mínimos. Sin embargo, el hecho de ampliar las características de los centros ha permitido obtener datos relevantes que nos han ayudado a analizar con mayor precisión la situación actual.

Más de un $30 \%$ de los colegios profesionales no tienen contemplado como servicio para sus colegiados un centro de documentación o unidad de información que facilite las posibilidades de acceso a la información bibliográfica a sus colegiados; es cierto que muchos de ellos tendrán la posibilidad de acceder a la información científica a través de sus centros de trabajo. A pesar de ello, los colegios profesionales no deberían relegar a un segundo plano garantizar una formación continuada a sus miembros, mediante la creación de buenas bibliotecas o centros de documentación.

\subsection{Identificación de la unidad de información}

Sería importante la existencia de una política de sistemas de información que potenciara la creación de unidades de información en el seno de las organizaciones colegiales, quizá la inexistencia de unas normas o recomendaciones al respecto provoca que la sensibilización en este terreno sea menor.

La escasa o nula identificación de la unidad de información, diferenciada de la organización a la que pertenece, pone de manifiesto esta carencia. 


\subsection{Fondos bibliográficos}

A pesar de encontrarnos en la llamada era de la información electrónica, donde se hace más hincapié en el acceso a la información que en la propiedad de la misma, las infraestructuras y sistemas de cooperación interbibliotecaria no están suficientemente desarrollados como para obviar la necesidad de disponer, todavía hoy, de unas colecciones que garanticen una cobertura mínima de las necesidades de información de determinados colectivos.

Únicamente cuatro centros disponen de una colección de más de 1.500 libros, la mayoría cuenta con pequeñas colecciones inferiores a 500 ejemplares. Sería importante analizar el contenido y origen de dichas colecciones, no sólo en lo que a cobertura cronológica, temática o idiomática se refriere, sino en cuanto a los criterios de inclusión de nuevo material, verificando que existan unos criterios de selección que garanticen que la colección sea de calidad y no un mero depósito de libros derivados de múltiples y descoordinadas procedencias. Muestra de ello nos la ofrecen los datos de la tabla II en la que podemos observar que el expurgo de material no se realiza en ninguno de los centros. Aunque la cantidad de libros en alguna biblioteca se podría considerar insuficiente, el aspecto en el cual se debería incidir es en la relevancia y adecuación del fondo a las necesidades de los usuarios, así como el nivel de actualización.

Respecto a las revistas especializadas, observamos que sólo el $30 \%$ de los centros estudiados dispone de una colección media de 250 títulos, los centros restantes cuentan con colecciones inferiores a 25 títulos. Si nos fijamos en las recomendaciones para bibliotecas médicas de tamaño pequeño, la tasa se sitúa en una colección inicial de 65 títulos (8), con lo que podemos ver que la cobertura de revistas especializadas es pobre en la mayoría de los centros.

Más del $50 \%$ de las bibliotecas estudiadas ofrece acceso a bases de datos, utilizando en igual medida el acceso en CD-ROM y el acceso a un servidor de bases de datos. Se han destacado como bases de datos más utilizadas MEDLINE e IME. Destaca la presencia de la base de datos CINHAL en colegios distintos de los de enfermería. Algunos de los centros ofrecen como servicio el acceso a MEDLINE y otras bases de datos a través de servidores gratuitos (PubMEd, etc.). Sería importante potenciar el acceso a bases de datos especializadas para cada uno de los colectivos.

\subsection{Procesos técnicos}

Podemos observar que de los centros que tienen catalogado su fondo, la mayoría lo tiene informatizado, a la vez que elabora catálogos impresos e informatizados de las colecciones de sus revistas. No es de sorprender que los centros que tienen su fondo informatizado a la vez sean los que aplican mayor número de procesos técnicos a los mismos, como puede ser la elaboración de listados.

A pesar de la informatización, la utilización de normas de catalogación es escasa, lo que lleva a pensar que muchos centros identifican la descripción bibliográfica con los procesos de catalogación propiamente dichos.

Se constata que ningún centro realiza tareas de expurgo. 


\subsection{Servicios}

En un 70\% de los centros el acceso y los servicios son restringidos a los colegiados, y en los casos de existir servicios sujetos a tarifas éstas se aplican por igual a todos los colectivos y tipos de usuarios.

\section{- Acceso a la información y consulta de bases de datos}

El servicio de búsquedas bibliográficas se ofrece en 8 de los centros analizados; vemos que el sistema de búsquedas asistidas es el medio predominante ( 7 centros), con lo cual podríamos pensar que nos alejamos de la tendencia ya estudiada en otros países (9) en la que se demuestra que los investigadores prefieren hacer ellos mismos las búsquedas bibliográficas. Los porcentajes obtenidos en la forma de acceso a la información (un $90 \%$ con ayuda del documentalista) nos ponen de manifiesto que la autonomía en la búsqueda de información dista mucho de consolidarse. Este hecho se justifica plenamente si observamos que únicamente un centro realiza formación de usuarios.

\section{- Servicio de referencia y Difusión Selectiva de Información}

Por lo que se refiere a los servicios de referencia y Difusión Selectiva de Información, los porcentajes obtenidos ponen de manifiesto la falta de estructuración y consolidación de los mismos.

\section{- Préstamo y obtención de documentos}

Los servicios de préstamo individual e interbibliotecario, así como la obtención de documentos, se ofrecen, en mayor porcentaje, en aquellos centros que disponen de su fondo informatizado. Estos datos ratifican la existencia de centros con una organización y oferta estructurada de acuerdo con los servicios básicos que ofrece cualquier biblioteca. Estos servicios, principalmente el de obtención de documentos, deberían potenciarse puesto que son imprescindibles para el usuario que ha realizado una búsqueda bibliográfica en bases de datos, a menos que ésta incluya el texto completo.

\section{- Cooperación}

El nivel de cooperación es bajo. Únicamente tres centros incluyen sus publicaciones periódicas en catálogos colectivos de publicaciones periódicas, ya sea a nivel de CCAA en el catálogo editado por la Coordinadora de Documentació Biomèdica de $\mathrm{Ca}$ talunya, o a nivel estatal en el Catálogo de Publicaciones Periódicas en Bibliotecas de Ciencias de la Salud: C-17. La necesidad de impulsar la cooperación y coordinación entre los centros se pone de manifiesto si analizamos el bajo número de centros que realizan tareas de intercambio y de gestión de duplicados.

\subsection{Equipamiento, tecnología y personal}

Podemos considerar como factores negativos, a la vez que significativos, que sólo un $50 \%$ de los centros cuente con medios de reprografía que permitan a los usuarios reproducir artículos pertenecientes a las colecciones de los centros. Mucho más desta- 
cables la carencia de personal especializado (sólo un $20 \%$ de los centros cuenta con personal cualificado como responsable de las unidades de información).

La conexión a Internet está disponible en el $80 \%$ de los centros; este hecho podríamos considerarlo positivo si tenemos en cuenta la importancia que este medio ya ha consolidado en el entorno de las bibliotecas y centros de documentación. A pesar de ello, la explotación del potencial que Internet supone para los centros de documentación no se refleja en la aplicación que los distintos centros hacen de ello, en lo que a servicios de biblioteca y documentación se refiere.

\section{Conclusiones}

El presente estudio pone de manifiesto la necesidad de estructurar y establecer unos servicios mínimos que deberían ofrecer los colegios profesionales a sus colegiados. El tamaño de la población objeto del presente estudio puede resultar insuficiente para establecer unas conclusiones sólidas y extensibles a otros centros, sin embargo ha permitido identificar una serie de deficiencias y diferencias entre centros de las mismas características expuestas en la discusión.

Las bibliotecas de los colegios profesionales presentan importantes carencias, derivadas probablemente de una falta de información y sensibilización ante los directivos de dichos centros y por la falta de asignaciones presupuestarias que dificultan la creación y mantenimiento de unos servicios mínimos.

Ante la irregularidad de la situación de los centros estudiados, se evidencia la necesidad de elaborar unas recomendaciones que sirvan de orientación para la creación de bibliotecas y/o centros de documentación de los colegios profesionales que garanticen a los profesionales unos servicios documentales de calidad, que les solucionen sus necesidades de información bibliográfica, independientemente de la situación laboral en la que se encuentren, a la vez que contribuyan a consolidar una red de bibliotecas en ciencias de la salud sólida. En este sentido, sería importante tener en consideración las recomendaciones incluidas en el proyecto SINAPSIS (5).

Queda abierta una línea de trabajo, centrada en los servicios que ofrecen los colegios profesionales, que sirva para potenciar unos servicios de información y documentación completos, con la máxima calidad posible.

\section{Referencias bibliográficas}

1. BRAVO JIMÉNEZ, R. Importancia de la documentación e información científica en la toma de decisiones clínicas. Actas de las VI Jornadas de Información y Documentación en Ciencias de la Salud. 1996, noviembre 15-17, Madrid

2. TREDINNICK, F. A. Small Colleges and Associations. New Directions for Higher Education 1983, vol 11 n. ${ }^{\circ} 2$, p. $85-90$

3. DALRYMPLE, P. W.; SCHERRER, C. S. Tools for improvement: a systematic analysis and guide to accreditation by the JCAHO. Bulletin of the Medical Library Association 1998, vol 86, n." 1, p. 10-16.

4. LÁZARO Y DE MERCADO, P.. et al. Las bibliotecas de Ciencias de la Salud en España: situación actual y propuestas de futuro (Presentación del Proyecto de Investigación CICYT INF-94-1551-E). Libro de actas de las VII Jornadas de Información y Documentación en Ciencias de la Salud. 1997, noviembre 6-8, Granada: ASABIME. 
5. GRUPO DE INVESTIGACIÓN EN BIBLIOTECAS DE SALUD. Las bibliotecas de Ciencias de la Salud en España: situación actual y propuestas de futuro. Madrid: Instituto de Salud Carlos III. 1997.

6. RODRfGUEZ DEL CASTILLO MARTÍN, M. Las bibliotecas de hospital en España ante las necesidades de información de los profesionales sanitarios. Medicina Clínica. 1998; vol. $110,{ }^{\circ} 14$, p. $543-547$.

7. UBIETO ARTUR, I., SÁNCHEZ CASABÓN, M. I., UBIETO ARTUR, A. P. El sistema de información aragonés: centros y servicios de información y documentación. Zaragoza: Departamento de Educación y Cultura, 1996.

8. R. HILL, D. Brandon/Hill selected list of books and journals for the small medical llibrary. Bulletin of the Medical Library Association 1999; vol 87, n. ${ }^{\circ} 2$, p. 145-169.

9. COLAINNI, L.A. A multidisciplinary approach to health information. I Congresso Regional de Informaçao em Ciencias da Sáude. Sao Paulo: BIREME. 1992. 